\title{
Enchimento de sementes em linhas quase-isogênicas de trigo com presença e ausência do caráter "stay-green"(1)
}

\author{
Simone Alves Silva(2), Fernando Irajá Félix de Carvalho ${ }^{(3)}$, Jorge Luís $\mathrm{Nedel}^{(3)}$ \\ Pedro Jacinto Cruz ${ }^{(2)}$, Silmar Teichert Peske ${ }^{(3)}$, Daniel Simioni ${ }^{(4)}$ e Adeliano Cargnin ${ }^{(4)}$
}

\begin{abstract}
Resumo - O caráter "stay-green" (permanência da cor verde nas plantas) tem sido utilizado para reduzir a senescência em trigo, resultando no aumento funcional da área foliar, e, conseqüentemente, na elevação da taxa e da duração do enchimento da semente. O objetivo deste trabalho foi analisar a taxa de acúmulo de matéria seca e a duração do período de enchimento da semente de linhas quase-isogênicas de trigo diferenciadas quanto à presença e ausência do caráter "stay-green". O experimento foi conduzido no Centro Experimental da Palma, Pelotas, RS. Foram utilizadas quatro linhas quase-isogênicas: $\mathrm{F}_{6}-\mathrm{SG}$ e $\mathrm{F}_{6}$-SZ, no ano de 2000 e $\mathrm{F}_{7}$-SG e $\mathrm{F}_{7}$-SZ no ano 2001, com cinco repetições. O caráter "stay-green" permitiu maior deposição de reservas na semente na fase próxima do ponto de maturidade físiológica. A duração do período de enchimento da semente esteve associada ao acúmulo de matéria seca na semente, promovendo um maior peso médio da semente. Assim, o progresso do caráter taxa de enchimento da semente poderá ser obtido por meio de seleções de plantas com maior peso médio da semente.
\end{abstract}

Termos para indexação: Triticum aestivum, matéria seca, peso da semente, senescência.

Seed filling in near-isogenic lines of wheat with presence and absence of the stay-green trait

Abstract - The stay-green trait has been used as an alternative to reduce the senescence in wheat, resulting in an effective functional increase of the leaf area, and an increase in the rate and duration of the seed filling period. The objective of this work was to analyse the rate of dry matter accumulation and the duration of the seed filling period of near-isogenic lines of wheat differentiated by the presence and absence of the stay-green trait. The experiment was conducted in the Experimental Center of Palma, in Pelotas, State of Rio Grande do Sul, in Brazil. Four near-isogenic lines were used: $\mathrm{F}_{6}-\mathrm{SG}$ and $\mathrm{F}_{6}-\mathrm{SZ}$ in the year of 2000 and $\mathrm{F}_{7}-\mathrm{SG}$ and $\mathrm{F}_{7}-\mathrm{SZ}$ in the year 2001, with five replications. The stay-green trait allowed a higher deposition of plant reserves in the seed, during the phase close to the point of physiological maturity. The duration of the seed filling period in the evaluated genotypes was associated to the dry matter accumulation in the seed. Promoting a larger weight is a trait responsible for the selection of genotypes with high rates of seed filling

Index terms: Triticum aestivum, dry matter, seed weight, senescence.

(1) Aceito para publicação em 15 de janeiro de 2003

(2) Universidade Federal da Bahia, Escola de Agronomia, Dep. de Fitotecnia, Campus Universitário, CEP 44380-000 Cruz das Almas, BA. E-mail: sas@ufba.br, cruzpj@ufba.br

(3) Universidade Federal de Pelotas (UFPel), Fac. de Agronomia Eliseu Maciel, Dep. de Fitotecnia, Caixa Postal 354, CEP 96010-160 Pelotas, RS.

E-mail: carvalho@ufpel.tche.br,jlnedel@ufpel.tche.br, peske@ufpel.tche.br

(4) UFPel. Bolsista do CNPq. E-mail: simioni@ufpel.tche.br, cargnin@ufpel.tche.br

\section{Introduç̃o}

O caráter "stay-green" tem sido utilizado como alternativa para promover redução da senescência em trigo (Triticum aestivum L.) (Silva, 1999; Silva, et al., 2000) em razão da maior permanência da área verde de folhas e colmos e conseqüente aumento no período de enchimento até a total formação da semente.

A produtividade do trigo tem sido limitada principalmente pelo produto final reprodutivo que é o grão 
(Slafer \& Andrade, 1991; Rodrigues, 2000). O período que corresponde ao crescimento da espiga próximo à antese até o desenvolvimento total da semente é de grande importância. Maior disponibilidade de assimilados próximos à antese pode representar mais flores férteis e, conseqüentemente, sementes em maior número e tamanho, com maior capacidade de formar grãos cheios (Slafer et al., 1994; Rodrigues et al., 1998; Rodrigues, 2000). Assim, uma vez determinado o número de sementes por espiga, o rendimento de sementes passa a depender da taxa de acúmulo da matéria seca e da duração do período de enchimento.

O enchimento da semente começa nas espiguetas centrais e progride até as basais e distais da inflorescência (Slafer et al., 1994; Rodrigues, 2000). Nesse momento, a área foliar verde tem grande importância como tecido fotossintetizante ativo, proporcionando maior partição dos assimilados, no enchimento da semente.

O efeito da temperatura na duração do período de enchimento e crescimento da semente tem sido bastante discutido. Segundo Rodrigues (2000), a ocorrência de baixas temperaturas retarda o desenvolvimento e aumenta o período de crescimento da semente, enquanto altas temperaturas provocam, além de redução no período de crescimento da semente, diminuição sensível de rendimento. Isso ocorre porque as temperaturas elevadas intensificam o movimento de assimilado da folha bandeira para a espiga, mas, por causa do aumento da taxa da respiração, não aumentam, necessariamente, a taxa de acúmulo de matéria seca (Gebeyehou et al., 1982; Bruckner \& Frohberg, 1987). Segundo Sanford (1985) e Nedel et al. (1999), a elevação da temperatura aumenta moderadamente a taxa de enchimento da semente, enquanto a duração do enchimento é drasticamente reduzida.

O colmo e as folhas permanecem verdes ("staygreen") até o completo enchimento da semente, que corresponde ao período da antese até a maturidade fisiológica, o que constitui estratégia eficiente na potencialização da disponibilidade de assimilados para a espiga e, conseqüentemente, na elevação do rendimento de sementes, principalmente o componente peso médio da semente.

O objetivo deste trabalho foi analisar a taxa de acúmulo de matéria seca e a duração do período de enchimento da semente de linhas quase-isogênicas de trigo diferenciadas somente quanto à presença $\mathrm{e}$ ausência do caráter "stay-green".

\section{Material e Métodos}

Nos anos de 1999 a 2001, utilizando duas épocas de semeadura por ano, estação quente e fria, em condições de campo e telado, foram feitos os avanços de gerações a partir do cruzamento de genótipos TB 188 (A-1TB-2TB1TB-901F-400F-902F-1TB-1TB-1TB-1TB) e TB 438 (D-1TB-1TB-1TB-1TB-1TB). Por meio de autofecundações até obter a geração $\mathrm{F}_{6}$, no ano 2000 , e $\mathrm{F}_{7}$ no ano 2001 , foram obtidas linhas quase-isogênicas, que diferiam entre si quanto à ausência e presença do caráter "staygreen". As linhas $\mathrm{F}_{6}-\mathrm{SG}$ e $\mathrm{F}_{7}-\mathrm{SG}$ correspondem às quaseisogênicas que apresentam o caráter "stay-green" e $\mathrm{F}_{6}-\mathrm{SZ}$ e $\mathrm{F}_{7}$-SZ as que não apresentam o caráter "stay-green". A ausência desse caráter determina a maturação sincronizada

As linhas quase-isogênicas foram semeadas nos dias 9 e 29 de outubro de 2000 e 2001, respectivamente, no campo do Centro Experimental da Palma (CEP), da Faculdade de Agronomia Eliseu Maciel da Universidade Federal de Pelotas, RS, para avaliação da taxa de enchimento da semente, duração do período de enchimento de sementes em dias e graus-dia acumulados e o peso da semente na maturidade fisiológica.

A unidade experimental era composta de três fileiras de 3 m de comprimento, preparadas com aração e gradagem. A densidade de semeadura foi de 160 sementes viáveis por $\mathrm{m}^{2}$. As dimensões de cada parcela foram de 3,0x0,9 m, com espaçamento de $0,3 \mathrm{~m}$ entre linhas. A semeadura foi feita manualmente e após a emergência, entre os estádios de afilhamento e alongamento, aplicou-se $\mathrm{N}$ na forma de uréia (Reunião..., 1999). Foi feito o controle de invasoras, com capinas manuais, além do controle de fungos na parte aérea com o fungicida Folicur.

Os procedimentos utilizados para aferir os caracteres avaliados foram adotados por Nedel et al. (1999). Por ocasião da antese, marcaram-se 80 espigas de diferentes plantas, no afilho principal, que apresentassem na região central extrusão recente das anteras, para que todas ficassem no mesmo estádio de desenvolvimento. Foram coletadas amostras de cinco espigas marcadas e colhidas a intervalos menores (dois dias) nas primeiras colheitas, iniciando no quinto dia após a antese e em períodos maiores (cinco dias) nas últimas colheitas. As espigas foram secadas em estufa com circulação de ar, à temperatura de $60^{\circ} \mathrm{C}$ por 48 horas. Nas sementes do terço médio da espiga de cada 
amostra foram determinados o número e o peso seco, para o cálculo do peso médio da semente.

A maturidade fisiológica foi estimada como a fase na qual se observou o máximo peso médio da semente. Em cada linha quase-isogênica obteve-se a relação entre dias após a antese e peso médio da semente, mediante a equação: $\mathrm{Y}=\mathrm{a}+\mathrm{bt}+\mathrm{ct}^{2}+\mathrm{dt}^{3}$,

em que: Y é o peso da matéria seca da semente (mg); $a, b$, c e d são os coeficientes de regressão; e t é o tempo, em dias, após a antese. O ponto de máximo acúmulo de matéria seca, que determina o ponto de maturidade fisiológica, foi alcançado quando a semente deixou de acumular matéria seca no t-ésimo dia após a antese e seu peso mantinhase constante.

O cálculo dos graus-dia (GD) foi realizado conforme Schneider et al. (1987), pela fórmula:

$\mathrm{GD}=(1 / 5)\left[\mathrm{T}_{9 \mathrm{~h}}+\mathrm{TM}+\mathrm{Tm}+\left(2 \mathrm{~T}_{21 \mathrm{~h}}\right)\right]-\mathrm{Tb}$,

em que: GD é o graus-dia; $\mathrm{T}_{9 \mathrm{~h}}$ e $\mathrm{T}_{21 \mathrm{~h}}$ são as temperaturas às $9 \mathrm{~h}$ e $21 \mathrm{~h}$, respectivamente; TM e Tm são as temperaturas máxima e mínima ocorridas no dia; e $\mathrm{Tb}$ é a temperatura base do trigo, que, segundo Mota (1989), poderia ser igual a $5^{\circ} \mathrm{C}$. A duração, em dias, do período de enchimento da semente, foi determinada do início da antese até atingir o ponto de maturidade fisiológica; a duração do período de enchimento da semente, em graus-dia acumulados (GDA), foi obtida pelo somatório dos GDA da antese à maturidade fisiológica.

A taxa de enchimento da semente (TE) expressa em matéria seca (mg) por GDA foi obtida pela divisão do peso da matéria seca (PMS) pelo número de sementes obtidas no terço médio da espiga (NTME) na maturidade fisiológica e, posteriormente, pelos GDA durante o período entre a antese e a maturidade fisiológica, como demonstrado a seguir:

\section{$\mathrm{TE}=\mathrm{PMS} / \mathrm{NTME} / \mathrm{GDA}$.}

O estudo do comportamento das diferenças entre o grupo "stay-green" e o grupo maturação sincronizada, obtidas entre os períodos de enchimento da semente, foi realizado por regressão polinomial

\section{Resultados e Discussão}

Houve diferença significativa em relação a todos os caracteres comparados entre as linhas quaseisogênicas "stay-green" ( $\mathrm{F}_{6}-\mathrm{SG}$ e $\left.\mathrm{F}_{7}-\mathrm{SG}\right)$ e as de maturação sincronizada $\left(\mathrm{F}_{6}-\mathrm{SZ}\right.$ e $\left.\mathrm{F}_{7}-\mathrm{SZ}\right)$ dentro de cada ano, respectivamente 2000 e 2001 (Tabela 1). Isto indica que a presença do caráter "stay-green" nessas duas linhas quase-isogênicas determinou variabilidade em relação aos caracteres avaliados.
A duração, em dias, da antese à maturidade fisiológica em 2000 foi superior à do ano $2001 \mathrm{em}$ ambas as linhas quase-isogênicas, ou seja, a $\mathrm{F}_{6}$-SG necessitou de maior número de dias para atingir a maturidade fisiológica (42 dias) do que a $\mathrm{F}_{6}$-SZ (32 dias). Também pode ser verificado proporção de valores no ano 2001, com magnitudes diferentes e inferiores, isto é, a linha quase-isogênica $\mathrm{F}_{7}-\mathrm{SG}$ manteve um período de enchimento mais longo (35 dias), e a $\mathrm{F}_{7}$-SZ atingiu a maturidade fisiológica com 30 dias. Essa diferença pode ser explicada por causa das condições distintas entre os dois ambientes avaliados; no ano 2000, o estádio entre a antese e a maturidade fisiológica ocorreu entre 9 de outubro e 16 de novembro e em 2001 entre 29 de outubro e 5 de dezembro, período este em que as plantas ficaram expostas a temperaturas médias mais elevadas.

Sofield et al. (1997) e Rodrigues (2000) também constataram que o aumento da temperatura causou redução no período de enchimento da semente. Entretanto, a taxa de enchimento de $\mathrm{F}_{6}-\mathrm{SG}$ e de $\mathrm{F}_{7}-\mathrm{SG}$ foi superior à de $\mathrm{F}_{6}-\mathrm{SZ}$ e de $\mathrm{F}_{7}-\mathrm{SZ}$, dentro de cada ano. A contribuição do caráter peso da semente na maturidade fisiológica (PSMF) foi expressiva sobre o elevado GDA na obtenção de uma maior taxa de acúmulo de matéria seca na semente, pois, com o aumento do GDA, é esperada redução na taxa de enchimento (Sanford, 1985). Uma taxa elevada em curto período de enchimento da semente resulta em baixo peso da semente na maturidade fisiológica

Tabela 1. Duração do período da antese à maturidade fisiológica (PAMF) e de graus-dia acumulados (GDA), taxa de acúmulo de matéria seca na semente, peso médio da semente estimado na maturidade fisiológica (PSMF) das linhas quase-isogênicas de trigo com o caráter "stay-green" $\left(\mathrm{F}_{6}-\mathrm{SG}\right.$ e $\left.\mathrm{F}_{7}-\mathrm{SG}\right)$ e sem o caráter "stay-green" com maturação sincronizada ( $\mathrm{F}_{6}-\mathrm{SZ}$ e $\mathrm{F}_{7}$-SZ), em dois anos. Pelotas, RS, 2001 ${ }^{(1)}$.

\begin{tabular}{lcccc}
\hline $\begin{array}{l}\text { Linha quase- } \\
\text { isogênica }\end{array}$ & $\begin{array}{c}\text { PAMF } \\
\text { (dias) }\end{array}$ & GDA & $\begin{array}{c}\text { Matéria seca } \\
(\mathrm{mg} / \mathrm{sem} / \mathrm{GDA})\end{array}$ & $\begin{array}{c}\text { PSMF } \\
(\mathrm{mg})\end{array}$ \\
\hline & & & 2000 \\
$\mathrm{~F}_{6}-\mathrm{SG}$ & $42 \mathrm{a}$ & $584,92 \mathrm{a}$ & $0,073 \mathrm{a}$ & $42,63 \mathrm{a}$ \\
$\mathrm{F}_{6}-\mathrm{SZ}$ & $32 \mathrm{~b}$ & $433,72 \mathrm{~b}$ & $0,061 \mathrm{~b}$ & $35,37 \mathrm{~b}$ \\
\hline & & & 2001 & \\
$\mathrm{~F}_{7}-\mathrm{SG}$ & $35 \mathrm{a}$ & $474,36 \mathrm{a}$ & $0,051 \mathrm{a}$ & $29,14 \mathrm{a}$ \\
$\mathrm{F}_{7}-\mathrm{SZ}$ & $30 \mathrm{~b}$ & $406,04 \mathrm{~b}$ & $0,045 \mathrm{~b}$ & $26,10 \mathrm{~b}$ \\
\hline
\end{tabular}

(1)Médias seguidas de mesma letra, dentro de anos, não diferem entre si a $5 \%$ de probabilidade pelo teste $\mathrm{F}$. 
(Aude et al., 1994). Entretanto, pode ser observado no presente trabalho elevada taxa de enchimento da semente, mesmo com alto valor de GDA. Para Bruckner \& Frohberg (1987), taxa de enchimento elevada também pode ser obtida mesmo com elevado GDA, desde que ocorra alto valor no peso da semente.

Valores elevados de GDA, correlacionados com a alta duração do período de enchimento, em dias, e com o peso médio da semente da antese à maturidade fisiológica, também foram obtidos por Bruckner \& Frohberg (1987) e Aude et al. (1994). Com isso, o aumento do período de enchimento da semente poderá proporcionar elevados valores no peso da semente, pois, segundo Wiegand \& Cuellar (1991), a taxa de acúmulo de matéria seca está associada, mais freqüentemente, a fatores genéticos e o período de enchimento da semente, aos fatores de ambiente. Assim, aumentar o peso da semente pelo incremento do período de enchimento não parece ser a melhor estratégia na obtenção de maiores rendimentos em ambientes com temperaturas elevadas (Bruckner \& Frohberg, 1987).

A variação genética na taxa de enchimento e peso médio da semente foi obtida por Nedel et al. (1999). Segundo esses autores, tal resposta é indicativo de provável associação entre os dois caracteres e deve ser levada em consideração, visto que é possível selecionar genótipos com maior taxa de enchimento de sementes sem aumentar o período de enchimento.

Uma vez que o período da antese à maturidade fisiológica é considerado o estádio mais crítico da espécie e sujeito às condições adversas do ambiente, é desejável manter a planta mais vigorosa e com maior poder de fotoassimilação sem estender demasiadamente o período de enchimento total da semente. As linhas providas do caráter "stay-green" requerem acúmulo maior de graus-dia para atingir o estádio de maturidade fisiológica do que as linhas com maturação sincronizada, embora ambas atingem o estádio de colheita em igual período (Tabela 1). Esse resultado permitiu destacar a eficiência deste caráter no enchimento da semente com menor tempo para a colheita.

O acúmulo de matéria seca na semente entre a antese e a maturidade fisiológica, em razão do número de dias após a antese, é mostrado nas Figuras 1 e 2. A equação de regressão polinomial de 3 o grau foi a que melhor se ajustou em todas as situações avaliadas, com coeficientes de determinação $\left(\mathrm{r}^{2}\right)$ superiores a 0,99 . A linha $\mathrm{F}_{6}-\mathrm{SG}$ necessitou de 42 dias e a $\mathrm{F}_{6}-\mathrm{SZ}$ de 32 dias para atingir peso de semente estimado de 42,63 mg e 35,57 mg na maturidade fisiológica, respectivamente (Figura 1). O maior período utilizado pela linha $\mathrm{F}_{6}-\mathrm{SG}$ para atingir o ponto de maturidade resultou em maior peso médio da semente. Desta forma, presume-se que o peso médio da semente está também associado ao maior tempo de enchimento da semente (Bruckner \& Frohberg, 1987; Aude et al., 1994). Entretanto, em relação aos genótipos avaliados, a seleção de elevadas taxas de acúmulo de matéria seca (Tabela 1) implica incremento de duração da taxa de enchimento, pois maior período de enchimento da semente poderá conferir maior capacidade de adaptação a estresses de ambiente que possam ocorrer, resultando em maior estabilidade de rendimento em genótipos com essa característica (Bruckner \& Frohberg, 1987; Aude et al., 1994).

O comportamento das linhas diferenciou-se em relação à duração de enchimento da semente, em dias, da antese à maturidade fisiológica (Figura 2). Houve redução no período mais quente (novembro e dezembro), ocasionando menor duração de GDA e menor taxa de enchimento. Isto resultou no menor peso médio da semente em ambas as linhas, embora a linha com o caráter "stay-green" ( $\left.\mathrm{F}_{7}-\mathrm{SG}\right)$, permanecesse superior na estimativa do peso final no ponto de maturidade (Figura 2).

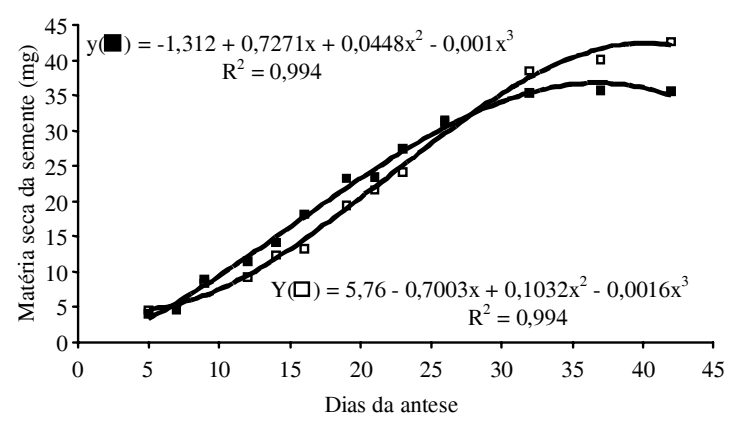

Figura 1. Acúmulo de matéria seca e período de enchimento da semente da antese à maturidade fisiológica em linhas quase-isogênicas de trigo com o caráter "stay-green" $\left(\mathrm{F}_{6}-\mathrm{SG}, \square\right)$ e sem o caráter "stay-green" com maturação sincronizada $\left(\mathrm{F}_{6}\right.$-SZ, $\left.\mathbf{\square}\right)$, no ano de 2000. 


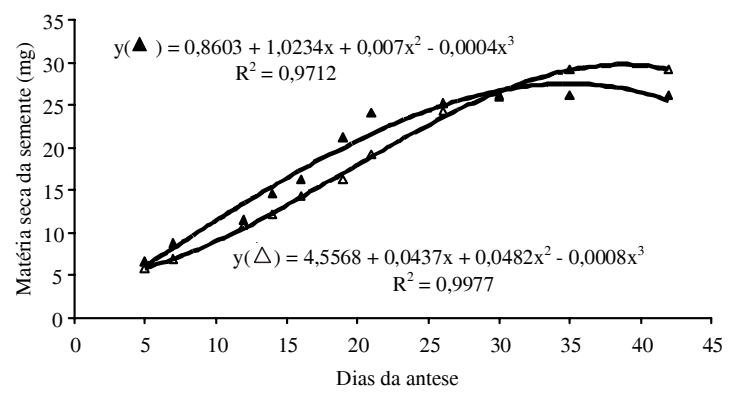

Figura 2. Acúmulo de matéria seca e período de enchimento da semente da antese à maturidade fisiológica em linhas quase-isogênicas de trigo com o caráter "stay-green" $\left(\mathrm{F}_{7} \mathrm{SG}, \triangle\right)$ e sem o caráter "stay-green" com maturação sincronizada $\left(\mathrm{F}_{7}-\mathrm{SZ}, \boldsymbol{\Delta}\right)$, no ano de 2001

As linhas quase-isogênicas com comportamento de maturação sincronizada, na fase inicial após o estádio de antese, produziram maior acúmulo de matéria seca, alcançando o ponto de maturidade fisiológica de forma mais precoce (Figuras 1 e 2). Essa manifestação fenotípica é almejada, desde que o peso final seja compensado por uma maior taxa de enchimento. Nas linhas quase-isogênicas "stay-green" ocorreu o inverso, ou seja, a fase inicial acumulou menor matéria seca, compensada na fase próxima à maturidade, quando ocorreu maior deposição de reservas na semente e, conseqüentemente, maior acréscimo do peso médio da semente.

\section{Conclusões}

1. Linhas "stay-green" apresentam maior eficiência no desenvolvimento do ciclo, alongando a fase de enchimento da semente e diminuindo o período para a senescência da planta.

2. A duração do período de enchimento da semente está associada ao maior acúmulo de matéria seca da semente.

3. O caráter "stay-green" propicia maior deposição de reservas na semente próximo à fase de maturidade fisiológica, que é crítico no aumento do peso médio da semente.

4. A ausência do caráter "stay-green" determina a redução do período de enchimento da semente e, conseqüentemente, menor peso médio da semente na maturidade fisiológica.
5. A utilização dos graus-dia acumulados permite classificar os genótipos quanto à precocidade da duração da taxa de enchimento no período da antese à maturidade fisiológica.

\section{Referências}

AUDE, M. I. S.; MARCHESAN, E.; MAIRESSE, L. A. S.; BISOGNIN, D. A.; CIMAR, R. J.; ZANINI, W. Taxa de acúmulo de matéria seca e duração do período de enchimento de grãos de trigo. Pesquisa Agropecuária Brasileira, Brasília, v. 29, n. 10, p. 1533-1539, out. 1994.

BRUCKNER, P. L.; FROHBERG, R. C. Rate and duration of grain fill in spring wheat. Crop Science, Madison, v. 27, n. 3 , p. $451-455,1987$.

GEBEYEHOU, G.; KNOT, D. R.; BAKER, R. J. Rate and duration of grain filling in durum wheat cultivates. Crop Science, Madison, v. 22, n. 2, p. 337-340, 1982.

MOTA, F. S. da. Agrometeorologia do trigo no Brasil. Campinas: Sociedade Brasileira de Agronomia, 1989. 122 p.

NEDEL, J. L.; GONZÁLEZ, C. N. E. M.; PESKE, S. T Variação e associação de características ligadas à formação do grão de genótipos de trigo. Scientia Agricola Piracicaba, v. 56, n. 4, p. 1255-1260, 1999

REUNIÃO DA COMISSÃO SUL-BRASILEIRA DE PESQUISA DE TRIGO, 31., 1999, Passo Fundo. Recomendações... Passo Fundo: Embrapa-CNPT, 1999. 86 p.

RODRIGUES, O. Manejo de trigo: bases ecofisiológicas. In: CUNHA, G. R.; BACALTCHUK, B. Tecnologia para produzir trigo no Rio Grande do Sul. Porto Alegre: Assembléia Legislativa do Rio Grande do Sul, 2000. p. 120-169. (Série Culturas - Trigo)

RODRIGUES, O.; LHAMBY, J. C. B.; DIDONET,A. D.; MARCHESE, J. A.; SCIPIONI, C. Efeito da deficiência hídrica na produção de trigo. Pesquisa Agropecuária Brasileira, Brasília, v. 33, n. 6, p. 839-846, jun. 1998.

SANFORD, D. A. van. Variation in kernel growth characters among soft red winter wheats. Crop Science, Madison, v. 25, n. 4, p. 626-630, 1985.

SCHNEIDER, R. M.; BURIOL, G. A.; HELDWEIN,A. B Temperatura base e soma térmica do subperíodo semeadura emergência das cultivares de soja. Revista do Centro de Ciências Rurais, Santa Maria, v. 17, n. 4, p. 229-308, 1987.

Pesq. agropec. bras., Brasília, v. 38, n. 5, p. 613-618, maio 2003 
SILVA, S. A. Estimativa da herança do caráter "staygreen” em genótipos de trigo hexaplóide. 1999. 56 f. Dissertação (Mestrado em Fitomelhoramento) - Universidade Federal de Pelotas, Pelotas, 1999

SILVA, S. A.; CARVALHO, F. I. F.; CAETANO, V. R.; OLIVEIRA, A. C.; CIMBRA, J. L. M.; VASCONCELLOS, N. J. S.; LORENCETTI, C. Genetic basis of stay-green trait in bread wheat. Journal of New Seeds, Binghamton, v. 2, p. 55-68, 2000.

SLAFER, G. A.; ANDRADE, F. H. Changes in physiological attributes of the dry matter economy of breed wheat (Triticum aestivum) through genetic improvement of grain yield potential at different regions of the world Euphytica, Wageningen, v. 58, p. 37-49, 1991.
SLAFER, G. A.; SATORRE, E. H.; ANDRADE, F. H. Increases in grain yield in bread wheat from breeding and associated physiological changes. In: SLAFER, G. A. (Ed.) Genetic improvement of field crops. New York: M. Dekker, 1994. p. 1-68.

SOFIELD, I.; EVANS, L. T.; COOK, M. G.; WAEDLAW, I. F. Factors influencing rate and duration of grain filling in wheat. Australian Journal of Plant Physiology, Collingwood, v. 4, p. 785-797, 1997.

WIEGAND, C. L.; CUELLAR, J. A. Duration of grain filling and kernel weight of wheat as affected by temperature. Crop Science, Madison, v. 21, n. 1, p. 95-101, 1991. 